\title{
Assignment of genetic linkage maps to diploid Solanum tuberosum pachytene chromosomes by BAC-FISH technology
}

\author{
Xiaomin Tang • Jan M. de Boer • \\ Herman J. van Eck • Christian Bachem • \\ Richard G. F. Visser • Hans de Jong
}

Received: 22 April 2009 / Accepted: 20 August 2009 / Published online: 23 September 2009

(C) The Author(s) 2009. This article is published with open access at Springerlink.com

\begin{abstract}
A cytogenetic map has been developed for diploid potato (Solanum tuberosum), in which the arms of the 12 potato bivalents can be identified in pachytene complements using multicolor fluorescence in situ hybridization (FISH) with a set of 60 genetically anchored bacterial artificial chromosome (BAC) clones from the RHPOTKEY BAC library. This diagnostic set of selected BACs (five per chromosome) hybridizes to euchromatic regions and corresponds to well-defined loci in the ultradense genetic map, and with these probes a new detailed and reliable pachytene karyotype could be established. Chromosome size has been estimated both from microscopic length measurements and from 4',6-diamidino-2-phenylindole fluorescencebased DNA content measurements. In both approaches, chromosome 1 is the largest $(100-115 \mathrm{Mb})$ and chromosome 11 the smallest (49-53 Mb). Detailed measurements of mega-base-pair to micrometer ratios have been obtained for chromosome 5, with average
\end{abstract}

Responsible Editor: Jiming Jiang.

X. Tang · J. M. de Boer $\cdot$ H. J. van Eck $\cdot$ C. Bachem •

R. G. F. Visser

Wageningen UR Plant Breeding, Wageningen University and Research Center,

6708 PB Wageningen, The Netherlands

H. de Jong $(\bowtie)$

Laboratory of Genetics, Wageningen University, P.O. Box 309, 6700 AH Wageningen, The Netherlands e-mail: hans.dejong@wur.nl values of $1.07 \mathrm{Mb} / \mu \mathrm{m}$ for euchromatin and $3.67 \mathrm{Mb} / \mu \mathrm{m}$ for heterochromatin. In addition, our FISH results helped to solve two discrepancies in the potato genetic map related to chromosomes 8 and 12. Finally, we discuss the significance of the potato cytogenetic map for extended FISH studies in potato and related Solanaceae, which will be especially beneficial for the potato genome-sequencing project.

Keywords potato (Solanum tuberosum) . fluorescence in situ hybridization · cytogenetic map . pachytene karyotype

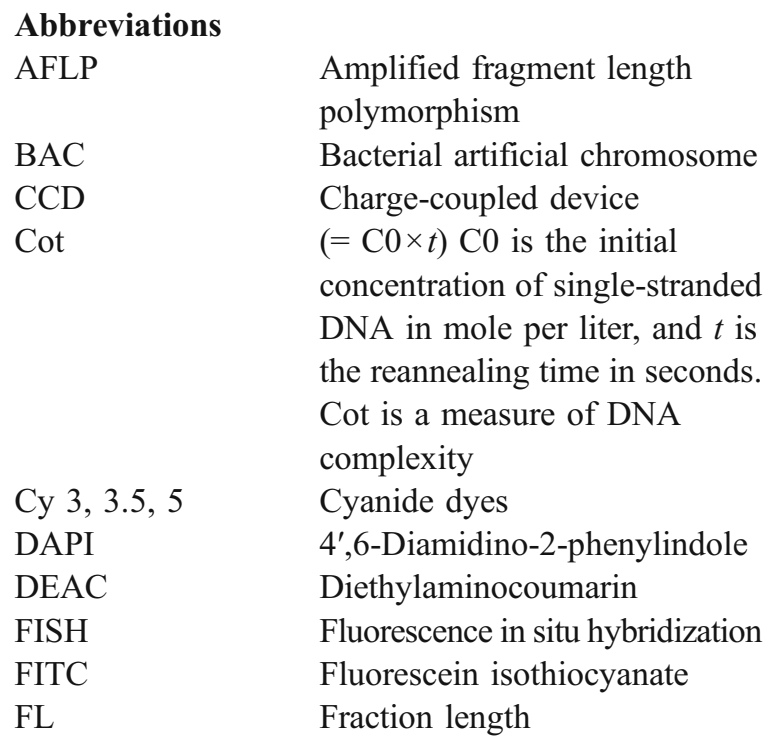




\begin{tabular}{|c|c|}
\hline NOR & Nucleolar-organizing region \\
\hline PGSC & Potato Genome-Sequencing \\
\hline & Consortium \\
\hline RH & $\begin{array}{l}\text { Paternal potato genotype used } \\
\text { in this study }\end{array}$ \\
\hline RHU & $\begin{array}{l}\text { Unidentified linkage group in } \\
\text { the } \mathrm{RH} \text { genotype }\end{array}$ \\
\hline $\mathrm{SH}$ & $\begin{array}{l}\text { Maternal potato genotype used } \\
\text { in this study }\end{array}$ \\
\hline TCC & Total cell complement \\
\hline UHD genetic map & Ultrahigh-density genetic map \\
\hline
\end{tabular}

\section{Introduction}

Potato (Solanum tuberosum), the third largest crop plant for food in the world, is a member of the Solanaceae, a plant family which includes many other economically important species, such as tomato, eggplant, petunia, tobacco, and pepper. The haploid potato genome comprises 12 chromosomes, corresponding to a total DNA size of about $840 \mathrm{Mb}$ (Bennett et al. 1997). Cultivar improvement in potato remains a timeconsuming process, and the genetic gains achieved over the last century are fairly small due to the complex genetics of this tetraploid outbreeder. It is therefore expected that development of potato genomics will be of great importance to support commercial breeding and cultivation.

For studying the inheritance of important agronomic traits in potato, several genetic maps have been created. Most of these maps were based on crosses between diploid lines (Bonierbale et al. 1988; Gebhardt et al. 1991; van Eck et al. 1995), although tetraploid maps are in use as well (Bradshaw et al. 2004). More recently, an ultrahigh-density (UHD) genetic map composed of approximately 10,000 amplified fragment length polymorphism (AFLP) markers was developed (van Os et al. 2006), which is most likely the densest map for a plant species ever constructed. The genetic positions of the centromeres were determined in this map by using half-tetrad analysis in a $4 x-2 x$ cross population of potato (Park et al. 2007). Considerable advances have also been made with genome-wide gene expression analysis, through microarray studies, expressed sequence tag sequencing, and serial analysis of gene expression (Nielsen et al. 2005; Rensink et al. 2005; Kloosterman et al. 2008).
As a major follow-up to these genetic and expressional characterizations of the potato genome, an international PGSC has been launched in 2006 (Visser et al. 2009; http://www.potatogenome.net/), which aims to elucidate the complete genomic DNA sequence of potato by 2010 . This sequencing project builds on a genomic DNA library of 78,000 bacterial artificial chromosome (BAC) clones derived from the paternal RH89-039-16 parent (hereafter referred to as clone RH) of the potato UHD genetic map. Currently, fingerprints of this BAC library have been assembled into a physical map of over 7,000 contigs (Borm 2008), of which 1,600 seed contigs are anchored to AFLP markers in the UHD map. This physical map forms the scaffold for the sequencing of the potato genome.

In contrast to the rapid progress of genetic and molecular studies of the potato genome, only limited progress has been achieved in the cytogenetic characterization of the potato genome. Very few heterochromatic or euchromatic features in the potato genome have been defined at the DNA sequence level. To date, the large amounts of genetic and sequence data have been only sparsely related to potato chromosome maps or structures. The chromosome morphology of the 12 potato pachytene bivalents was described previously (Yeh and Peloquin 1965; Ramanna and Wagenvoort 1976; Wagenvoort 1988). Yet, it was difficult to identify the chromosomes unequivocally by only morphological features, and none of these early pachytene karyotypes of potato were aligned with the currently available genetic or physical maps.

Several papers have reported to map DNA sequences by in situ hybridization technologies on mitotic metaphase chromosomes of potato (Visser et al. 1988; Wagenvoort et al. 1994; Fuchs et al. 1996; Dong et al. 2000). However, these somatic chromosome complements provide only low mapping resolution and few details regarding chromatin structure. Instead, meiotic pachytene chromosomes in pollen mother cells are much longer (de Jong et al. 1999) and display a clear heterochromatic-euchromatic differentiation, making them highly appropriate for high-resolution mapping of single-copy and repetitive sequences on chromosomes.

Application of BAC fluorescence in situ hybridization (BAC-FISH) on pachytene complements can contribute significantly to the construction of physical maps and to map-based cloning projects by confirming the physical locations of markers on the linkage groups, by resolving the order of closely linked 
markers, by identifying the heterochromatin and euchromatin borders, and by determining the locations of repetitive and single-copy DNA sequences (Lapitan et al. 1997; Zhong et al. 1999; Fransz et al. 2000; Cheng et al. 2001a; Kulikova et al. 2001; Islam-Faridi et al. 2002; Zhao et al. 2002; Wang et al. 2006b; Koo et al. 2008; Szinay et al. 2008). In potato, pachytene BAC-FISH has already been applied successfully to the characterization of chromosome 6 (Iovene et al. 2008; Tang et al. 2008).

In this report, we present the first genome-wide cytogenetic map of potato using a five-color BACFISH protocol on pachytene complements. With this map, several applications are demonstrated, including the construction of a standard pachytene karyotype which is integrated with the genetic and physical map of potato, a major repair in the chromosome 12 linkage map, anchor point verifications in the potato physical map, and comparisons between genetic, cytogenetic, and physical distances in the potato genome. The results reported in this FISH study provide a foundation for a detailed molecular cytogenetic characterization of the potato genome.

\section{Materials and methods}

For preparing cell spread preparations, we used anthers of the diploid potato genotypes G254 (Hermsen et al. 1978) and RH89-039-16 (Rouppe van der Voort et al. 1997). BAC clones were from the RHPOTKEY BAC library of genotype RH89-039-16 (Borm 2008). Genetic mapping information for the selected BAC clones (Table 1) was retrieved from the ultradense genetic map (van Os et al. 2006; http:// www.plantbreeding.wur.nl/potatomap/) and the potato physical map (Borm 2008, unpublished results). Genetic mapping information for BAC clone RH195L21 on chromosome 12 was kindly provided by Gerard Bijsterbosch (Wageningen UR Plant Breeding). The telomeric DNA probe pAtT4 cloned from Arabidopsis thaliana (Richards and Ausubel 1988) was also included in some of the FISH analyses.

\section{FISH and image capturing}

Pachytene chromosome preparations were made as described by Zhong et al. (1996a) with few minor modifications. Cot100 fractions of potato genomic
DNA for suppressing repetitive sequences in some BACs from hybridization to the chromosomes were prepared according to Chang et al. (2008) and Tang et al. (2008). BAC DNA was isolated using a standard alkaline extraction and directly labeled with one of the five fluorescent nucleotides: Cy3-dUTP, Cy3.5dCTP, Cy5-dUTP (GE Healthcare, Sweden), fluorescein-12-dUTP (fluorescein isothiocyanate, FITC), and diethylaminocoumarin (DEAC)-5-dUTP (PerkinElmer Inc.). The labeling methods followed the protocols of Amersham Bioscience (GE Healthcare, Sweden). FISH was performed according to the protocol of Zhong et al. (1996b). Slides were examined under a Zeiss Axioplan 2 Imaging Photomicroscope equipped with epifluorescence illumination, with filter sets for 4',6-diamidino-2-phenylindole (DAPI), DEAC (blue), FITC (green), Cy3 (orange), Cy3.5 (red), and Cy5 (far-red) fluorescence. Selected images were captured by a Photometrics Sensys $1,305 \times 1,024$ pixel $\mathrm{CCD}$ camera. Image processing and FISH signal thresholding were performed with the Genus Image Analysis Workstation software (Applied Imaging Corporation). DAPI images were sharpened separately with a $7 \times 7$ Hi-Gauss high-pass spatial filter to accentuate minor details and heterochromatin differentiation of the chromosomes. The FISH signals of the different fluorescence images were combined with the DAPI image in a multichannel stack. DAPI images were displayed in gray, and the other fluorescence images were pseudocolored mostly corresponding to the original colors of the fluorescing dyes. Brightness and contrast of the composite images were further improved with Photoshop CS4 (Adobe).

Data analysis

Prior to FISH, we analyzed a separate set of DAPIstained pachytene bivalents for measuring the size and positions of euchromatin and heterochromatin. We used ImageJ (http://rsb.info.nih.gov/ij) for measurements and for straightening chromosomes (plug-in of Kocsis et al. 1991). The absolute lengths of the chromosomes were also converted into relative lengths, as percentage of total cell complement (TCC).

For a direct comparison between genetic and physical positions of BACs, the cytological position of each BAC clone was converted into fraction length (FL) according to Cheng et al. (2001a). In this method, the total physical length of each pachytene 
Table 1 Genetic and physical locations of potato BAC clones

\begin{tabular}{|c|c|c|c|c|c|c|}
\hline $\begin{array}{l}\text { Chromosomal } \\
\operatorname{arm}^{\text {a }}\end{array}$ & AFLP marker & $\begin{array}{l}\text { Genetic position } \\
\text { (bin) }\end{array}$ & $\begin{array}{l}\text { Selected } \\
\text { BAC }\end{array}$ & $\begin{array}{l}\text { Physical location } \\
(\mathrm{FL})^{\mathrm{c}}\end{array}$ & Number $^{\mathrm{d}}$ & $\begin{array}{l}\text { Neighbor BACs' distance } \\
\text { comparison }\end{array}$ \\
\hline $1 \mathrm{~S}$ & EAACMCAG_211 & 7 & RH083J12 & $4.70 \pm 0.63$ & 10 & 7 bins/4.7 FL \\
\hline $1 \mathrm{~S}$ & EAGTMAGG_391 & 10 & RH078E17 & $8.53 \pm 1.14$ & 5 & 3 bins $/ 3.83 \mathrm{FL}$ \\
\hline $1 \mathrm{~L}$ & EAACMCAA_191.4 & 20 & RH092C10 & $47.79 \pm 2.40$ & 10 & $10 \mathrm{bins} / 39.26 \mathrm{FL}$ \\
\hline $1 \mathrm{~L}$ & EACAMCTG_271H & 47 & RH158N18 & $61.63 \pm 2.17$ & 10 & 27 bins/13.84 FL \\
\hline $1 \mathrm{~L}$ & EACTMCTA_205 & 101 & RH096H03 & $98.30 \pm 1.48$ & 10 & 54 bins/36.67 FL \\
\hline $2 \mathrm{~L}$ & EAGCMAAC_360 & 2 & RH075N07 & $14.70 \pm 4.63$ & 2 & 2 bins/14.7 FL \\
\hline $2 \mathrm{~L}$ & EAAGMCGA_432.8 & 7 & RH095G12 & $22.72 \pm 1.62$ & 5 & 5 bins/8.02 FL \\
\hline $2 \mathrm{~L}$ & EACAMAGC_150H & $13-14$ & RH174A15 & $29.71 \pm 1.74$ & 4 & 6-7 bins/6.99 FL \\
\hline $2 \mathrm{~L}$ & EAGAMACG_179 & 57 & RH076J19 & $57.42 \pm 0.92$ & 5 & 43-44 bins/27.71 FL \\
\hline $2 \mathrm{~L}$ & EACTMCAT_403.1H & 65 & RH055P13 & $61.49 \pm 0.64$ & 4 & $8 \mathrm{bins} / 4.07 \mathrm{FL}$ \\
\hline $3 \mathrm{~S}$ & EAAGMACC_433 & 9 & RH078O14 & $6.99 \pm 1.17$ & 3 & 9 bins/6.99 FL \\
\hline $3 \mathrm{~S}$ & EAACMCCA_261.7 & 13 & RH159O01 & $9.46 \pm 0.67$ & 2 & 4 bins/2.47 FL \\
\hline $3 \mathrm{~L}$ & EAGAMCAG_410.1 & 37 & RH079E02 & $47.43 \pm 1.06$ & 6 & 24 bins/37.97 FL \\
\hline $3 \mathrm{~L}$ & EAGAMCTC_245 & 52 & RH074E07 & $56.01 \pm 0.22$ & 2 & 15 bins $/ 8.58 \mathrm{FL}$ \\
\hline $3 \mathrm{~L}$ & EATGMCAT_364 & 79 & RH055M19 & $73.89 \pm 0.15$ & 2 & 27 bins/17.88 FL \\
\hline $4 \mathrm{~S}$ & EAACMCTC_297.6 & 1 & RH013K09 & $3.17 \pm 0.29$ & 3 & $1 \mathrm{bin} / 3.17 \mathrm{FL}$ \\
\hline $4 \mathrm{~S}$ & EAACMCAA_89.3 & 17 & RH162B09 & $8.90 \pm 0.58$ & 5 & 16 bins/5.73 FL \\
\hline $4 \mathrm{~S}$ & EAACMCGA_321.8 & 28 & RH097G17 & $17.25 \pm 1.59$ & 3 & 11 bins/8.35 FL \\
\hline $4 \mathrm{~L}$ & EACTMCTA_113.6 & 66 & RH095G10 & $81.14 \pm 2.24$ & 3 & 38 bins/63.89 FL \\
\hline $4 \mathrm{~L}$ & EAACMAGG_190H & 78 & RH191L17 & $94.82 \pm 2.07$ & 3 & 12 bins/13.68 FL \\
\hline $5 \mathrm{~L}$ & EAACMCAG_149.7 & 4 & RH081B09 & $3.61 \pm 0.46$ & 10 & 4 bins/3.61 FL \\
\hline $5 \mathrm{~L}$ & EAACMAGG_167 & 21 & RH095I08 & $12.37 \pm 1.19$ & 10 & 17 bins/8.76 FL \\
\hline $5 \mathrm{~L}$ & EAGAMACC_230 & 37 & RH076O08 & $21.40 \pm 1.77$ & 10 & 16 bins $/ 9.03 \mathrm{FL}$ \\
\hline $5 \mathrm{~S}$ & EACTMCTA_188.9H & 55 & RH089A21 & $61.89 \pm 1.32$ & 8 & 18 bins/40.49 FL \\
\hline $5 \mathrm{~S}$ & EAACMCTC_205 & 60 & RH044A21 & $68.60 \pm 1.16$ & 10 & 5 bins/6.71 FL \\
\hline $6 \mathrm{~S}$ & EATCMCTC_104.2 & 3 & RH026H24 & $2.50 \pm 0.35$ & 5 & 3 bins $/ 2.5 \mathrm{FL}$ \\
\hline $6 \mathrm{~S}$ & EAACMCCT_377 & 15 & RH069B12 & $9.09 \pm 0.69$ & 6 & 12 bins/6.59 FL \\
\hline $6 \mathrm{~L}$ & EACTMAGC_175 & 24 & RH075G13 & $37.18 \pm 1.52$ & 5 & 9 bins/28.09 FL \\
\hline $6 \mathrm{~L}$ & EAACMCTG_152.6 & $46-47$ & RH194M18 & $50.31 \pm 2.26$ & 5 & $22-23 \mathrm{bins} / 13.13 \mathrm{FL}$ \\
\hline $6 \mathrm{~L}$ & EACAMAGG_67 & 56 & RH054L23 & $60.29 \pm 1.45$ & 4 & 9-10 bins/9.98 FL \\
\hline $7 \mathrm{~L}$ & EACCMAGT_27H & 9 & RH075O19 & $5.16 \pm 0.83$ & 8 & $9 \mathrm{bins} / 5.16 \mathrm{FL}$ \\
\hline 7L & EACGMAGT_270 & 31 & RH078E15 & $10.81 \pm 1.35$ & 9 & 22 bins $/ 5.65 \mathrm{FL}$ \\
\hline 7L & EAAGMCAT_362 & 44 & RH170M01 & $18.46 \pm 0.70$ & 4 & 13 bins/7.65 FL \\
\hline $7 \mathrm{~L}$ & EAACMCAA_217rh & $48-49$ & RH093G10 & $21.33 \pm 1.50$ & 6 & 4-5 bins/2.87 FL \\
\hline $7 \mathrm{~S}$ & EAAGMCAT_231.9H & 77 & RH186I02 & $75.20 \pm 0.75$ & 8 & $28-29 \mathrm{bins} / 53.87 \mathrm{FL}$ \\
\hline $8 \mathrm{~S}$ & EACAMAGC_160rh & 7 & RH122L16 & $6.99 \pm 0.73$ & 8 & 7 bins/6.99 FL \\
\hline $8 \mathrm{~S}$ & EAAGMAGG_277.8 & 14 & RH055L21 & $13.72 \pm 1.60$ & 9 & 7 bins/6.73 FL \\
\hline $8 \mathrm{~L}$ & EAACMCCA_141 & 26 & RH184D07 & $54.14 \pm 2.75$ & 9 & 12 bins/40.42 FL \\
\hline $8 \mathrm{~L}$ & EACCMAAC_173 & 42 & RH122E19 & $71.39 \pm 3.29$ & 8 & 16 bins/17.25 FL \\
\hline $8 \mathrm{~L}$ & EAGAMACG_121 & 79 & RH127J02 & $89.33 \pm 1.35$ & 8 & 37 bins/17.94 FL \\
\hline $9 \mathrm{~S}$ & EACCMCAT_372 & 3 & RH135I22 & $4.39 \pm 0.65$ & 5 & 3 bins/4.39 FL \\
\hline $9 \mathrm{~S}$ & EAACMCTC_215.2H & 28 & RH061A13 & $20.89 \pm 1.89$ & 5 & 15 bins/16.50 FL \\
\hline 9L & EAACMCTT_255.6 & 34 & RH101N09 & $54.08 \pm 1.51$ & 7 & 6 bins/33.19 FL \\
\hline 9L & EAACMCAG_167.8 & 60 & RH168F09 & $71.99 \pm 1.22$ & 4 & 26 bins/17.91 FL \\
\hline
\end{tabular}


Table 1 (continued)

\begin{tabular}{|c|c|c|c|c|c|c|}
\hline $\begin{array}{l}\text { Chromosomal } \\
\operatorname{arm}^{\text {a }}\end{array}$ & AFLP marker & $\begin{array}{l}\text { Genetic position } \\
\text { (bin) }^{b}\end{array}$ & $\begin{array}{l}\text { Selected } \\
\text { BAC }\end{array}$ & $\begin{array}{l}\text { Physical location } \\
(\mathrm{FL})^{\mathrm{c}}\end{array}$ & Number $^{\mathrm{d}}$ & $\begin{array}{l}\text { Neighbor BACs' distance } \\
\text { comparison }\end{array}$ \\
\hline 9L & EAAGMACG_273H & 84 & RH079O06 & $82.35 \pm 1.29$ & 4 & 24 bins/10.36 FL \\
\hline $10 \mathrm{~L}$ & EAACMCAG_409.1 & 1 & RH049J10 & $5.14 \pm 0.66$ & 4 & $1 \mathrm{bin} / 5.14 \mathrm{FL}$ \\
\hline $10 \mathrm{~L}$ & EAACMCTT_143H & $25-27$ & RH048F15 & $13.18 \pm 0.96$ & 4 & $24-26 \mathrm{bins} / 8.04 \mathrm{FL}$ \\
\hline $10 \mathrm{~L}$ & EAACMCCA_222H & 34 & RH184D02 & $20.33 \pm 2.27$ & 4 & 7-9 bins/7.15 FL \\
\hline $10 \mathrm{~S}$ & EACAMCTG_80.2H & 76 & RH106M22 & $91.19 \pm 1.89$ & 4 & $42 \mathrm{bins} / 70.86 \mathrm{FL}$ \\
\hline $10 \mathrm{~S}$ & EAGTMCCA_221.1 & 86 & $\mathrm{RH} 178 \mathrm{~K} 07^{\mathrm{e}}$ & $97.31 \pm 1.16$ & 3 & $10 \mathrm{bins} / 6.12 \mathrm{FL}$ \\
\hline $11 \mathrm{~L}$ & EACAMCTT_252.5 & 7 & RH $204 \mathrm{G} 21^{\mathrm{e}}$ & $6.12 \pm 0.74$ & 2 & 7 bins/6.12 FL \\
\hline $11 \mathrm{~L}$ & EAAGMACC_206.5H & $25-27$ & RH097I18 & $16.74 \pm 1.15$ & 5 & $18-20 \mathrm{bins} / 10.62 \mathrm{FL}$ \\
\hline $11 \mathrm{~L}$ & EAACMCAT_1 & 41 & RH162O21 & $26.41 \pm 1.08$ & 5 & $14-16$ bins/9.67 FL \\
\hline $11 \mathrm{~S}$ & EACAMCTC_157.4rh & 68 & RH058F17 & $71.13 \pm 0.72$ & 5 & 27 bins/44.72 FL \\
\hline $11 \mathrm{~S}$ & EACAMCGA_262.5H & $82-83$ & RH042C12 & $75.38 \pm 0.48$ & 2 & $14-15$ bins/4.25 FL \\
\hline $12 \mathrm{~L}$ & EACAMACG_110 & 2 & RH106P06 ${ }^{\mathrm{e}}$ & $3.23 \pm 0.78$ & 9 & 2 bins $/ 3.23 \mathrm{FL}$ \\
\hline $12 \mathrm{~L}$ & EAGAMCTC_372 & 5 & RH016H06 & $6.97 \pm 1.14$ & 8 & 3 bins/3.74 FL \\
\hline $12 \mathrm{~L}$ & EACCMCAT_76 & 10 & RH183I02 & $10.93 \pm 1.01$ & 9 & 5 bins/3.96 FL \\
\hline $12 \mathrm{~L}$ & EACCMAAC_202 & 39 & RH043B17 & $20.58 \pm 1.77$ & 10 & 29 bins/9.65 FL \\
\hline $12 \mathrm{~S}$ & EAGAMCAG_116.5 & 90 & RH084C24 & $88.46 \pm 1.01$ & 10 & 51 bins/67.88 FL \\
\hline
\end{tabular}

${ }^{a}$ Arabic numerals, chromosome number; $\mathrm{S}$ and L, short and long arm of potato chromosomes. The numbering system was according to corresponding linkage groups (van Os et al. 2006)

${ }^{b}$ The genetic position was adopted from the ultradense RH genetic map (van Os et al. 2006). The genetic map consisted of bin segments that each represent one recombination event (equivalent to 0.77 -cM distance) that was observed in the $\mathrm{SH} \times \mathrm{RH}$ mapping population

${ }^{\mathrm{c}}$ Physical location FL was calculated as $(\mathrm{S} / \mathrm{T}) \times$ binT, where $\mathrm{S}=$ the distance in $\mu \mathrm{m}$ from the FISH hybridization site to the north end of the chromosome, $\mathrm{T}=$ the total length of the chromosome in micrometer, binT=the total bin value of the same linkage group (van Os et al. 2006)

${ }^{\mathrm{d}}$ The number of measurements

${ }^{\mathrm{e}}$ Ten BACs as cytogenetic markers were used to identify bivalents 3 to 12 , respectively, in pachytene cell complement

chromosome was divided into a number of FLs equal to the genetic length of the corresponding linkage group. For genetic lengths of the RH genetic map, we used bins, in which one bin equals about $0.77 \mathrm{cM}$, and converted the number of bins of that linkage group into the number of FLs per chromosome. For example, the length of linkage group RH1 is 101 bins, and hence the pachytene chromosome 1 is divided into $101 \mathrm{FLs}$. The physical locations of BACs were then calculated as $\mathrm{FL}=(S / T) \times$ binT, where $S=$ the distance in micrometer from the FISH hybridization site to the north end of the chromosome, $T=$ the total length of the chromosome in micrometer, and binT = the total bin value of the same linkage group.

Relative DAPI fluorescence intensities were measured with Image-Pro Plus 6.0 software by summing pixel intensities in the selected area of interest. Data were further processed in an MS Excel spreadsheet. For certain DAPI fluorescence measurements, we first calculated that $10 \mu \mathrm{m}$ corresponds to 236 pixels in the microscopic image, then picked randomly nine euchromatin or heterochromatin blocks of $10-\mu \mathrm{m}$ length each, and converted these into mega-base-pair per micrometer by taking the total sum of DAPI fluorescence intensity $\times$ $840 \mathrm{Mb} /$ total density sum of cell complement for euchromatin and for heterochromatin, respectively.

\section{Results}

Alignment of cytogenetic map and linkage groups of potato

We developed a cytogenetic map with full chromosome identification in potato, on the basis of selected BAC sets for each of the 12 potato chromosomes (Table 1) covering both short and long arms (except for chromosome 2 for which BACs cover only the 
long arm). These 60 BAC clones have been anchored to AFLP markers in all 12 linkage groups and are taken from physical map contigs in regions of the genetic map with a medium to high recombination rate. For each set of five BACs per chromosome, we labeled the clones with the five available fluorescent dyes and hybridized them simultaneously to produce a reference pattern of markers/marker bands. Most BACs were located in the euchromatic regions and produced distinct single fluorescence foci, even without Cot100 blocking, suggesting that they were poor in repeat sequences.

Two criteria were used to verify that the selected BACs hybridize according to their anchoring AFLP marker positions. Firstly, we checked if each group of five BACs from a particular linkage group indeed hybridized to the same chromosome (Fig. 1). Secondly, we made sure that the chromosomal positions of all 60 selected BACs correlated with their corresponding genetic AFLP marker positions (Table 1), especially with respect to their order on their maps.

The resulting reference FISH map was aligned with the ultradense genetic linkage map, and this comparison revealed the distance relations between the chromosomal positions of the BAC clones and genetic positions of the corresponding anchoring AFLP markers (Fig. 1). Marker intervals were compared between both maps, and some discrepancies were detected between genetic distances expressed in bins and chromosomal distances expressed in FLs (Table 1). Differences were particularly obvious for marker intervals spanning the pericentromere heterochromatin and the centromere regions. For instance, clones RH061A13 and RH101N09, which are most likely located close to the euchromatin and pericentromere heterochromatin boundaries on the short arm and long arm of chromosome 9, respectively, are separated by six bins in the genetic map, whereas their physical distance is 33.19 FL (Table 1), which is a 5.5-fold difference.

Towards a standard potato pachytene karyotype

We have constructed a standard pachytene karyotype for potato using five-color BAC-FISH signals in combination with the differential DAPI staining of euchromatin and heterochromatin regions. Chromosomes 1 and 2 are easily identifiable in the cell complement and do not require BAC painting: chromosome 1 is always the longest in the complement, and chromosome 2 contains the satellite with the conspicuous nucleolar-organizing region (NOR) in the short arm. The remaining chromosomes (3 to 12) were tagged with a set of ten BACs (indicated by superscripted $\mathrm{e}$ in Table 1) with known position on each of these chromosomes. These ten BACs were labeled with five different fluorescent dyes and used for FISH on selected slides with well-spread and clearly differentiated late pachytene complements. This procedure allowed the simultaneous identification of all 12 bivalents in a cell complement of genotype G254 (Fig. 2).

The length of each bivalent was measured in 16 cells (Fig. 2, Table 2). The longest chromosome 1 measures $81.73 \mu \mathrm{m}(13.70 \%$ TCC), whereas the shortest chromosome 11 is $37.71 \mu \mathrm{m}(6.30 \%$ TCC). Taking into account the lengths of all chromosomes in the cell complements, we found the order of the chromosomes from longest to smallest to be, respectively, chromosomes 1, 4, 3, 2 (including the rDNA section of the chromosome), 6, 9, 8, 7, 10, 12, 5, and 11. The constriction and often weaker-stained region in the brightly fluorescing pericentromere marks the position of the centromere and was used to determine short and long arms. Short arms of most chromosomes were aligned as expected to north arms in the ultradense genetic map, which fits the "short arm on top" convention. But for chromosomes 5, 7, 10, 11, and 12, the short arms were found to align with the south arms in the genetic map.

DAPI staining reveals a clear differentiation of heterochromatin domains in the pachytene complements of potato that resembles the heterochromatin patterns of aceto-carmine-stained pachytene complements (cf. Ramanna and Wagenvoort 1976) and Cbanded mitotic metaphase chromosomes (Pijnacker and Ferwerda 1984). All chromosomes have brightly fluorescing pericentromeres composed of a variable number of different-sized heterochromatin blocks (Fig. 2, Table 2), with a large heterochromatic satellite in chromosome 2 and minor blocks at most of the chromosome ends. We always compared heterochromatin differentiation before and after FISH, as hybridization conditions tend to lead to decrease chromatin stainability. On the basis of length measurements and DAPI fluorescence intensity of all heterochromatin and euchromatin segments (Table 2), we calculated that heterochromatin occupies approx- 
Fig. 1 Potato cytogenetic map and its alignment to the UHD genetic map of genotype RH. For each chromosome (white numbers), five BAC clones were selected (see Table 1) as cytogenetic markers and were hybridized to DAPIstained pachytene chromosomes by five-color FISH. The FISH images (right) were straightened and stretched to align with the corresponding genetic maps (left) for relative distance comparison. Each linkage group of the $\mathrm{RH}$ genetic map is divided into bin segments (small rectangles) of 0.77-cM length, in which the AFLP marker density is coded by a grayscale value. The black and dark gray bins have the highest marker densities. The centromere locations on the pachytene chromosomes are indicated by asterisks. The

corresponding genetic positions of the centromeres are marked according to Park et al. (2007), except for RH linkage group 3 (see text)
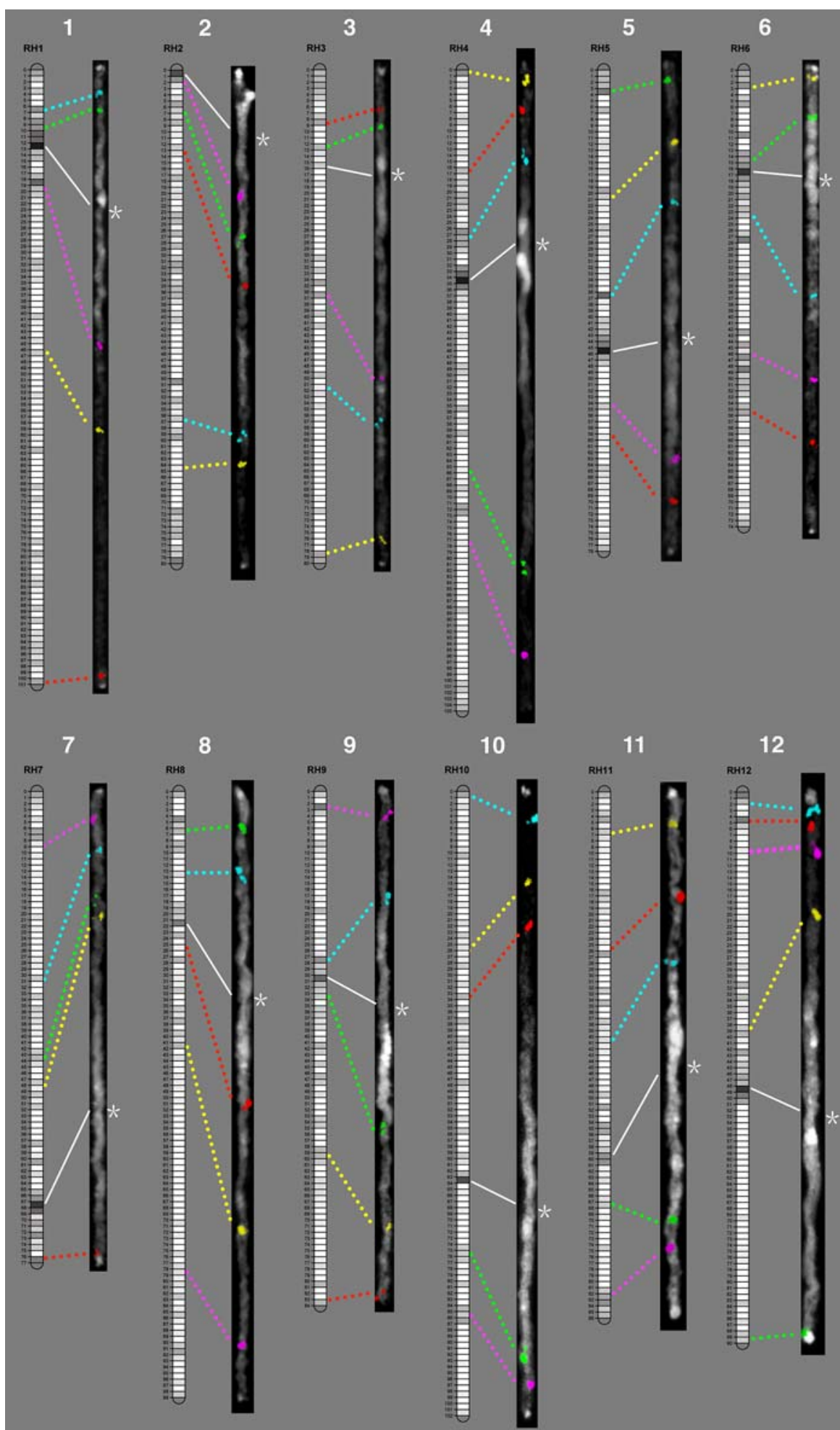

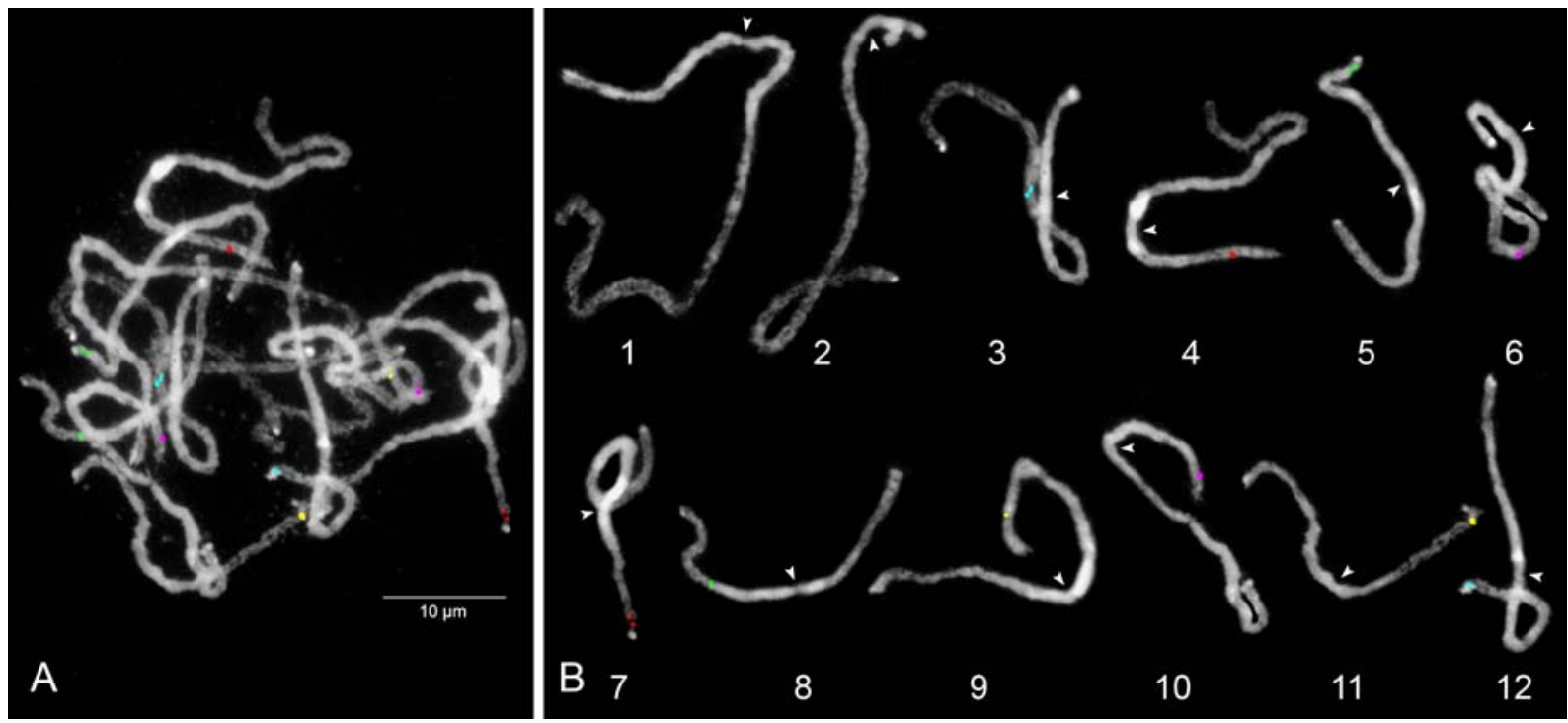

Fig. 2 Karyotyping of pachytene chromosomes of Solanum tuberosum cultivar G254. a The complement of pachytene chromosomes. The DAPI-stained chromosomes displayed brightly fluorescent pericentromere heterochromatin around the centromeres (primary constriction within the heterochromatic region). Five-color FISH with ten chromosome-specific

imately $32.8 \%$ of the TCC. The smallest chromosome 11 contains $5.86 \%$ of the DAPI fluorescence which equals $49.18 \mathrm{Mb}$ (of the total genome size of $840 \mathrm{MB}$ ), and the longest chromosome 1 is $11.9 \%$ and $99.82 \mathrm{Mb}$, respectively. Furthermore, we measured DAPI fluorescence for several randomly chosen regions of euchromatin or heterochromatin (see "Materials and methods") and calculated average DNA content values of $0.86 \pm 0.17 \mathrm{Mb} / \mu \mathrm{m}$ for euchromatin and $3.85 \pm 0.60 \mathrm{Mb} / \mu \mathrm{m}$ for heterochromatin, respectively. Considering that the heterochromatin percentage is $32.8 \%$ of the TCC, we estimated that $68.6 \%$ of the potato genome is in heterochromatin (i.e., $3.85 \times 32.8 \% /(0.86 \times 67.2 \%+3.85 \times 32.8 \%)=$ $68.6 \%)$. Further comparisons of length, arm ratio, and heterochromatin distribution features of pachytene chromosomes between G254 and RH89-039-16 (data not shown) support the concept that the two potato accessions have similar chromosome portraits and that G254 may serve as a representative karyotype of potato.

FISH characterization of physical map anchors

We have used the reference set of five landmark BACs for each chromosome to examine FISH
BACs allowed the identification of all 12 bivalents in one FISH experiment. b Bivalents were digitally separated from a. Chromosomes were ordered and numbered according to corresponding linkage groups and indicated by arrowheads at centromere position

locations of more selected BAC clones of the potato physical map, to ensure that the resulting maps are highly accurate and fit well with each other. The reliability of the AFLP anchor points was verified by hybridization to pachytene chromosomes for a total of $158 \mathrm{BAC}$ clones (including the 60 reference clones of the chromosome map). For 141 clones ( $\sim 89 \%)$, FISH positions corresponded well with their markers on the genetic map. Discrepancies were found for 13 BACs ( $\sim 8 \%$ ), of which ten clones displayed a single focus on another chromosome than predicted genetically. Eight of them were later found to have incorrect physical map anchors, whereas two were due to mistakes in clone culturing and tracking. The three remaining clones mapped in a position a few bins away from their expected marker positions, which could be explained by small inaccuracies in the genetic map. There were four more BACs $(\sim 3 \%)$ that bound to multiple chromosomal targets, including the pericentromere regions of other chromosomes, even with Cot100 blocking, indicating that these BACs have a very high percentage of repetitive sequences.

To further support sequencing activities exploring the physical size of the euchromatic regions, we were also keen on BACs close to or at the telomeres and euchromatin/heterochromatin borders. As shown in 


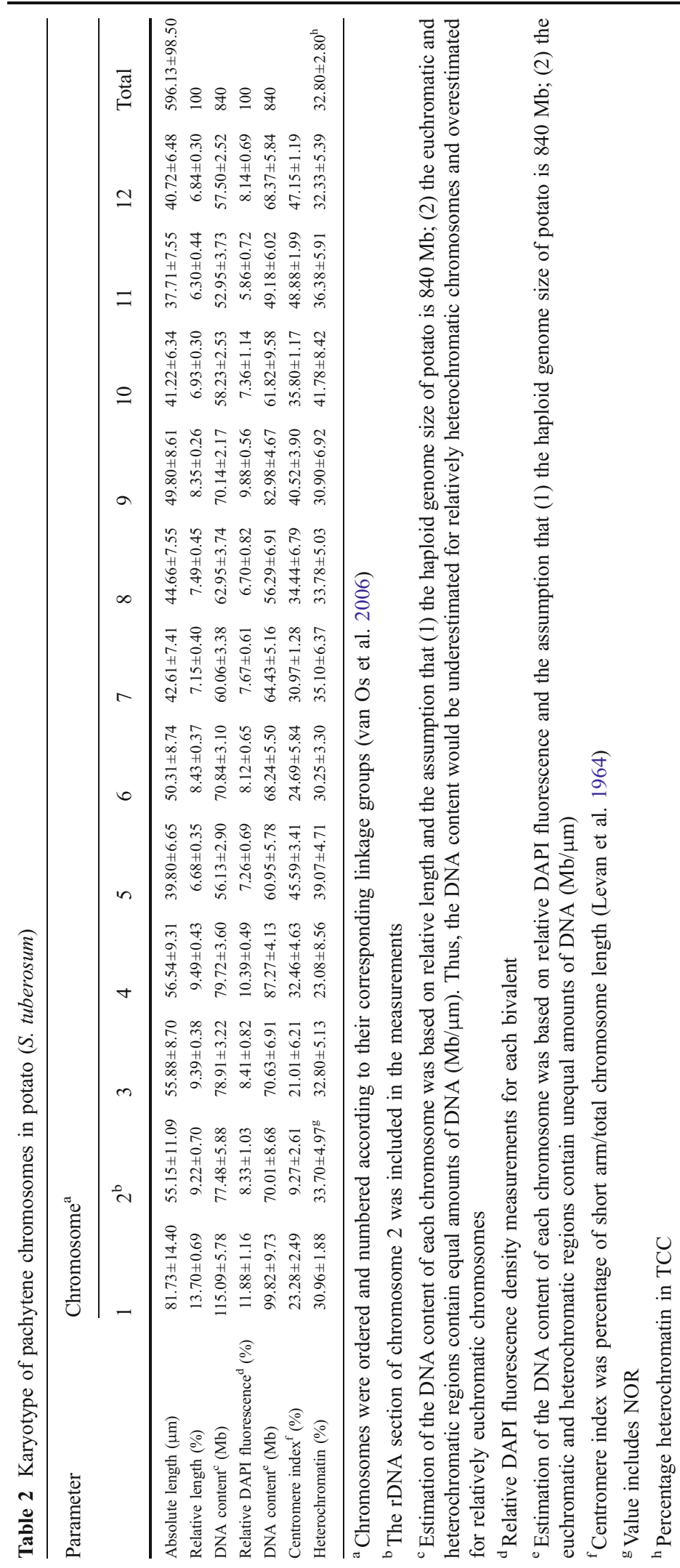




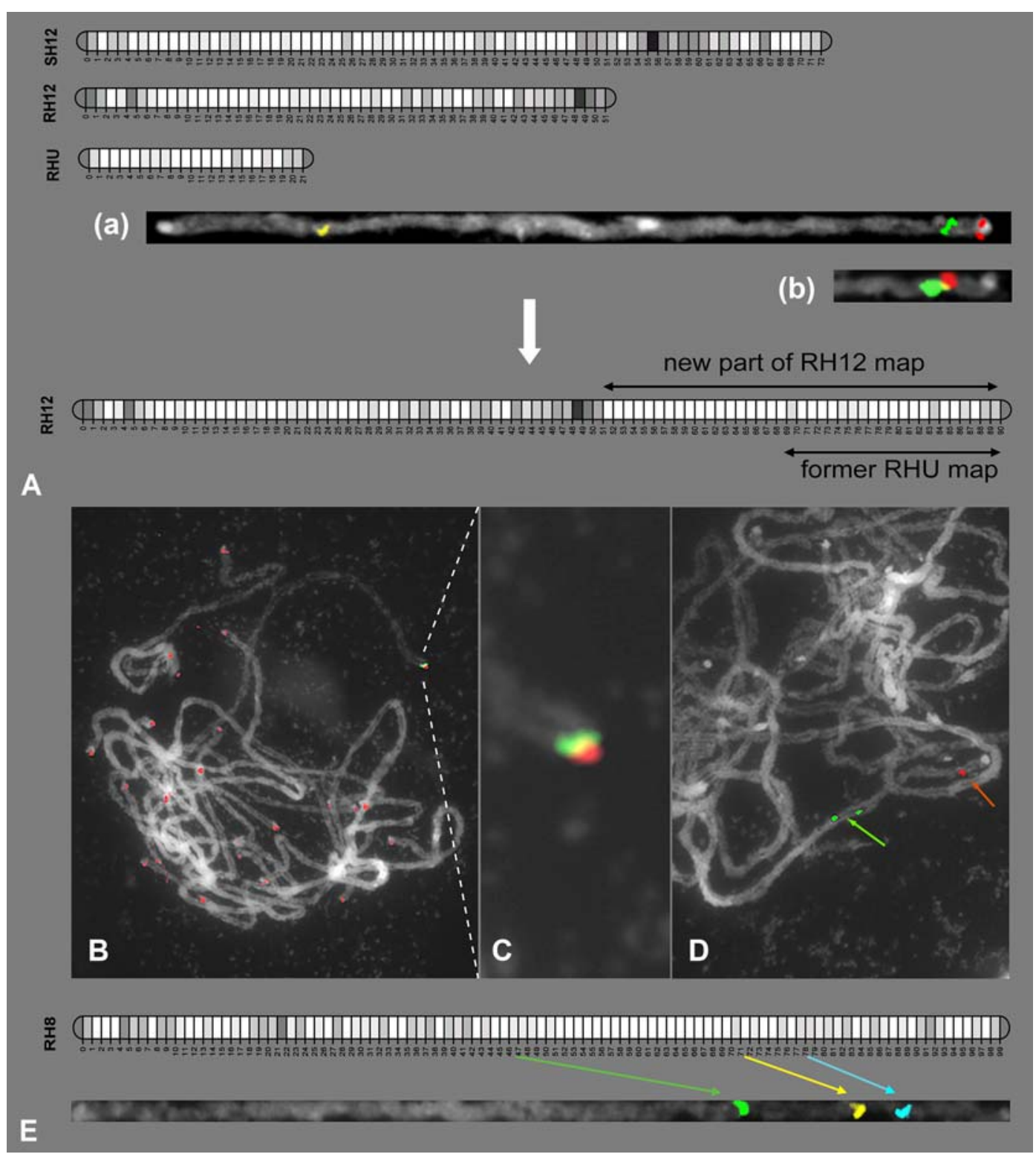

Fig. 3 a Placement of unassigned linkage group RHU at the end of chromosome 12, by which a new RH12 map was developed. (a) FISH mapping of two BAC clones (RH057E14, green; RH084C24, red) anchored by RHU markers and one BAC clone (RH043B17, yellow) from RH12 bin 39 on the straightened chromosome 12. (b) FISH mapping of BAC clone (RH057E14, red), anchored by an RHU marker, and BAC clone (RH195L21, green), which was mapped to bin 67 of the SH12 map, to the distal part of straightened chromosome 12. b,

Fig. 3d, RH061A13 was identified as a BAC clone at the border of euchromatin and pericentromere heterochromatin of the short arm of chromosome 9. An important assessment of the quality of a genetic linkage map is its physical coverage of the c One of the most distal BAC clones (RH106H24, green) from chromosome 1 was shown to partially overlap (indicated by yellow color) with telomeric repeats (pAtT4, red). d FISH mapping of two BAC clones from the euchromatic portion of chromosome 9 of which the BAC clone (RH061A13, green signal) bordered the pericentromere heterochromatin. e FISH mapping of three chromosome 8 BAC clones (RH168E16, green; RH048O11, yellow; RH127J02, blue) showed a large physical gap between RH168E16 and RH048O11

corresponding chromosome. BAC clone RH106H24 carries AFLP marker EAACMCAA_467, which anchors it to bin101 at the south end of potato linkage group 1 . The FISH signal from $\mathrm{RH} 106 \mathrm{H} 24$ partially overlapped with the signals derived from the Arabi- 
Fig. 4 BAC-FISH mapping resolution for chromosome 5 . a FISH mapping of $12 \mathrm{BAC}$ clones specific on chromosome 5 showed four gaps along the short arm of chromosome 5. b FISH mapping of BACs located at pericentromere heterochromatin region of chromosome 5. (a) FISH mapping of one BAC clone (RH132D05, green) anchored by a marker from putative centromere bin046. With excess amount of Cot100, it was hybridized at north heterochromatin very proximal to the centromere.

Two BAC clones

(RH076O08 and RH044A21, red signals) helped us to identify chromosome 5. (b) FISH mapping of two BAC clones (RH094I11, pink; RH048J07, red) anchored by markers from putative centromere bin046. BAC clone (RH075N11, blue) helped us to identify chromosome 5. (c) FISH mapping of three BAC clones (RH130M21, yellow; RH096H07, red; RH071D16, green) anchored by markers from putative centromere bin046. BAC clone (RH075N11, blue) helped us to identify chromosome 5

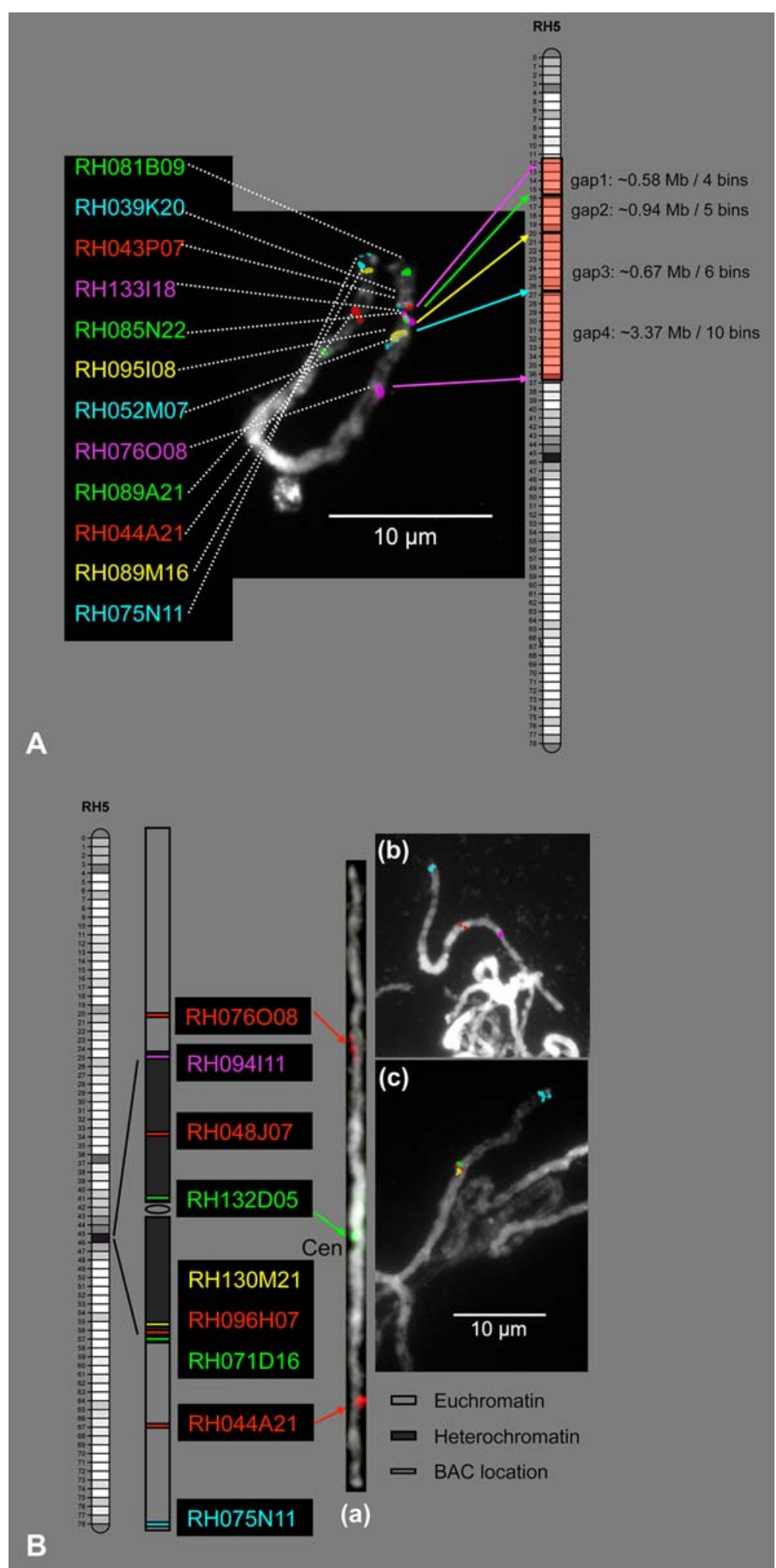


dopsis telomeric (TTTAGGG) DNA clone pAtT4 on pachytene chromosome 1 (Fig. $3 \mathrm{~b}, \mathrm{c}$ ). Thus, $\mathrm{RH} 106 \mathrm{H} 24$ is the BAC demarcating the end of the long arm of chromosome 1 and so marks the very end of its linkage map.

Major gap analysis on chromosomes 8 and 12

The FISH map was also used to solve the location of a previously unassigned linkage group of markers from the genetic map of genotype $\mathrm{RH}$, the so-called RHU linkage group (van Os et al. 2006). In comparison to the maternal linkage group 12 (SH12) of the UHD map, the south end of paternal linkage group 12 (RH12) seemed truncated at the markerdense pericentromeric bin051, since no continuation into a second genetic map arm was observed (Fig. 3a). Both the highly skewed marker segregation $(102: 21)$ at this end of the RH12 map and the unexpected shorter length suggested that this unassigned part of the RH map (RHU) might be the lacking arm of RH12. Attempts to construct a linkage group integrating RH12 with RHU failed with normal map construction software because the statistical power to detect linkage was severely affected by skewed marker segregations. Furthermore, the distance between the ends of both linkage groups suggested a gap of at least 19 bins. A new linkage map has been constructed, guided by graphical genotyping and retrieving the more severely skewed markers from bin051 of RH12 and from the group of unassigned markers. This manual merger of RHU to RH12 was highly speculative, and therefore we were keen to seek a cytogenetic confirmation of this genetic map. From each of the two BAC contigs of the potato physical map tagged by AFLP markers from RHU, we selected one BAC (RH057E14 and RH084C24) as probe for FISH mapping. FISH analysis showed that the two BACs were indeed located at the end of chromosome 12 (Fig. 3a). This confirmed that RHU was correctly merged to RH12 via a gap of at least 19 empty bins (i.e., 19 recombination events). Further confirmation of this merge came from the localization in the RHU region of clone RH195L21 (Fig. 3a), which has a PCR marker that was mapped to bin 67 of the of maternal chromosome 12 map (SH12). The new RH12 genetic map now has 90 bins and is included in Fig. 1. The centromere position of chromosome 12 , which is located in bin 49-51 of the RH map, is no longer considered to be telocentric, as proposed by Park et al. (2007), and is now shown to be submetacentric, on the basis of both the new genetic map and the karyotype analysis.

The second major gap in the linkage map of genotype $\mathrm{RH}$ is located in the long arm of chromosome 8, where an AFLP marker-free region of 19 bins $(14.6 \mathrm{cM})$ that is considered troublesome for further genome sequencing exists from bin052 to bin070 (Fig. 3e). By pachytene FISH mapping of the BACs RH168E16 (bin049) and RH048O11 (bin073) flanking this linkage gap, we measured a distance between these two FISH signals of $6.17 \pm 0.72 \mu \mathrm{m}$. Assuming an average euchromatin density of $0.86 \mathrm{Mb} / \mu \mathrm{m}$ (see above), the size of this gap was estimated at $5.3 \pm$ $0.6 \mathrm{Mb}$. Thus, this genetic gap was revealed as a large physical gap problematic to close with sequencing through BAC contig extension because of the scarcity of genetic markers in this region.

Quantification of euchromatin and heterochromatin regions in chromosome 5

The current BAC by BAC sequencing of chromosome 5 of potato genotype $\mathrm{RH}$ has made considerable progress, and draft sequence tiling paths are now available for preliminary analysis (unpublished results). In conjunction with this sequencing effort, FISH analyses have been performed with chromosome 5 BACs to gather more detailed information about the relations between genetic, cytogenetic, and physical distances in potato and to estimate gaps in the sequence tiling path.

In the euchromatin region of the long arm, one tiling path was identified which contains eight overlapping BACs from clone RH043P07 to clone RH133I18 and has a draft sequence length of $780 \mathrm{~kb}$. FISH of these two border BACs showed a local DNA density of $1.07 \mathrm{Mb} / \mu \mathrm{m}$ for euchromatin of chromosome 5 (Fig. 4a). Genetically, this tiling path covers two bins (bin010 to bin012) and therefore includes two recombination events, which gives a recombination density of $1.54 \mathrm{cM} / 780 \mathrm{~kb}=1.97 \mathrm{cM} / \mathrm{Mb}$. In the pericentromere heterochromatin region, a 2,300-kb sequence tiling path spans 28 overlapping BACs. It runs from RH130M21 to RH071D16 and RH096H07 in between at $900-\mathrm{kb}$ distance from RH071D16. The positions of the FISH signals were in agreement with the clone 
order in this tiling path (Fig. 4b (c)). The length of the FISH interval between RH130M21 and RH071D16 was on average $0.626 \mu \mathrm{m}$, which indicated a local DNA density of $3.67 \mathrm{Mb} / \mu \mathrm{m}$ for heterochromatin of chromosome 5. These estimates correspond well with the previously mentioned DAPI-based estimates of $0.86 \mathrm{Mb} / \mu \mathrm{m}$ for euchromatin and $3.85 \mathrm{Mb} / \mu \mathrm{m}$ for heterochromatin.

Next, we used the sequence density value of chromosome 5 to estimate the physical size of four gaps in the euchromatic sequence tiling path. No connection existed between the south end of a tilling path ending in clone RH095I08 (bin021) and the north end of an eight BAC cluster beginning from clone RH052M07 (bin027). The genetic distance of six bins suggested the presence of a large gap (gap3; Fig. 4a). However, FISH revealed that both clones were actually relatively close to each other, separated by only $0.67 \mathrm{Mb}$ (i.e., the distance between the two FISH signals observed from RH095I08 and RH052M07 was $0.62 \pm 0.14 \mu \mathrm{m}$, implying a physical distance of $0.62 \mu \mathrm{m} \times 1.07 \mathrm{Mb} / \mu \mathrm{m}=0.67 \mathrm{Mb}$ ). An opposite result was found for the distance between RH052M07 (bin027) and RH076O08 (bin037), which were separated by a larger genetic gap of ten bins, corresponding with a large physical gap of $3.37 \mathrm{Mb}$ (gap4; Fig. 4a). In the same way, gap1 and gap2 could be estimated at 0.58 and $0.94 \mathrm{Mb}$, respectively. Gap1 and gap3 indicated a higher recombination rate than gap2 and gap4, thus hinting at the fact that gap1 and gap3 might be associated with recombination hot spots (Fig. 4a).

The pericentromere region is characterized by a strong suppression of recombination as was shown for tomato by Sherman and Stack (1995), and hence genetic markers from this region cluster into extremely marker-dense bins on the UHD genetic map (van Os et al. 2006). In genotype $\mathrm{RH}$, the centromere of chromosome 5 is located in bin046 (Park et al. 2007). The AFLP markers from this bin are responsible for anchoring about $70 \%$ of the BAC contigs in the physical map of chromosome 5 (unpublished observations), suggesting that bin046 physically spans the larger part of this chromosome's DNA. We have explored the extent of bin046 on the cytogenetic map by hybridization with BAC clones that were mapped to this genetic region. Hybridization of the repetitive sequences in such BACs was effectively suppressed by unlabeled Cot 100 genomic
DNA (100 times the probe DNA concentration) to the hybridization mixture. Six BACs from bin046 produced well-defined FISH signals in the pericentromere region (Fig. 4b). The interval covered by these clones extended from RH094I11 in the long arm to RH071D16 in the short arm, confirming the continuity of bin046 in the heterochromatic region. RH094I11 was located far from RH048J07; RH048J07 was in the northern pericentromere block halfway to the centromere; RH132D05 was very close to the centromere but still in the pericentromere heterochromatin; the other three BACs RH130M21, RH096H07, and RH071D16 were in turn south of the centromere (Fig. 4b). From the microscopic measurement of the RH094I11RH071D16 interval, it is concluded that bin046 indeed occupies the heterochromatic region and that its length corresponds to approximately $40 \%$ of the length of pachytene chromosome 5 .

\section{Discussion}

Potato chromosome identification

Mitotic chromosomes of potato are small and similar in size, and most of them are difficult to identify morphologically without additional C-banding (Pijnacker and Ferwerda 1984). At pachytene, the chromosomes are much longer and show a differentiated heterochromatin pattern, providing more landmarks for identification (Ramanna and Wagenvoort 1976). But even so, not all pachytene chromosomes of potato are easily distinguished, since several of them share similar size, arm ratio, and heterochromatin distribution patterns.

The technique of chromosome identification with FISH using chromosome-specific BAC clones has been well demonstrated in many plant species. It avoids the limitations that are associated with classic methods of chromosome identification and is particularly useful for plant species with a large number of small chromosomes (Dong et al. 2000, 2001, 2005; Cheng et al. 2001b; Kim et al. 2002; Tek et al. 2004; Tang et al. 2007). In potato, BAC-FISH has already been used for identification of all 12 chromosomes in mitotic cells (Dong et al. 2001), while in pachytene cells, chromosomes have been identified from only two linkage groups (Iovene et al. 2008; Tang et al. 2008; Achenbach et al. 2009). 
In this study, we performed various five-color FISH experiments with chromosome-specific BACs in potato to provide a set of 60 cytogenetic markers for chromosome identification and high-resolution cytogenetic mapping in pachytene complements. The 12 chromosomes are identified according to their linkage group number in the UHD genetic map (Van Os et al. 2006). The chromosome numbering system in potato has been adopted from the highly syntenic tomato genome (Bonierbale et al. 1988; Gebhardt et al. 1991), where the chromosome numbers are based on the decreasing order of bivalent lengths observed at pachytene (Barton 1950).

Pachytene karyotype of the potato genome

We developed a potato karyotype that was based on five-color FISH labeling with a selection of only ten BAC clones that are anchored to linkage groups 3 to 12 of the UHD genetic map. This one-step hybridization allowed the simultaneous identification of all potato bivalents in a single pachytene complement.

We found that the descending order of pachytene chromosome lengths is $1,4,3,2$ (including the rDNA section of the chromosome), $6,9,8,7,10,12,5$, and 11, which differs from the chromosome order in tomato, as defined by Barton (1950). This difference may be caused by differences in the procedure of cell preparation, but it may also reflect true differences between the potato and the tomato genome. The short arms of chromosomes 5, 7, 10, 11, and 12 aligned with the south arms of their linkage groups in the UHD genetic map, which is not in accordance with genetic convention. For chromosomes 7, 10, and 12, this unusual alignment is caused by the UHD map, which has its marker order upside down for these three chromosomes. For chromosomes 5 and 11, however, the marker order of the UHD map follows the other existing potato maps. This means that the short arm in potato is homologous to the long arm in tomato and vice versa. This discrepancy may be caused by potential misidentification of the centromeres of tomato by Barton (1950) or by an inaccuracy of chromosome arm measurements in these metacentric chromosomes.

The cytological centromere in the pachytene chromosomes could in most cases be established and their positions aligned well with their genetic centromere positions (Park et al. 2007). The exception is the centromere on chromosome 3 , which is most proba- bly located in bin016 (Fig. 1) instead of bin035 (Park et al. 2007). However, centromeres in some chromosomes do not clearly display the characteristic lightly stained chromatin domains, as was also shown for rice where the centromere-specific probe RCS2 did not hybridize at some of what was considered the primary constrictions of these chromosomes (Cheng et al. 2001b). In those cases, a centromere-specific repeat will be needed to demonstrate unequivocally the position of the centromere. However, so far, no such repeat has been found.

The DAPI-stained heterochromatin distribution patterns in potato pachytene chromosomes were not as distinct as those of tomato. The transition between euchromatin and heterochromatin in potato was generally less sharp but more gradually defined than in the tomato chromosomes (Yeh and Peloquin 1965). The boundary of euchromatin and heterochromatin in potato could therefore only be defined with certainty when close comparisons were made with acetocarmine-stained complements and the DAPI-stained complements before the FISH treatment. So we chose chromosomes showing clear heterochromatin patterns for measurements to best avoid existing small variations as discussed above. Our measurements showed that the major part of the chromosomes consist of euchromatin (only $32.8 \%$ heterochromatin). These results are roughly in agreement with the values for tomato (35.8\%; Peterson et al. 1996) but differ strongly with other estimates of $24 \%$ (de Jong et al. 1999) and $14.6 \%$ for M. truncatula (Kulikova et al. 2001), and A. thaliana $7.4 \%$ for (Fransz et al. 1998). We also estimated that $31.4 \%$ of the potato genome lies in euchromatin and $68.6 \%$ of the potato genome lies in heterochromatin that is considered rich in repetitive sequences, largely transcriptionally inactive, although cloning and characterization of rice chromosome 8 centromere did reveal the presence of active genes in centromere and flanking heterochromatin sequences (Nagaki et al. 2004; Wu et al. 2004; Yan et al. 2005).

We presented two methods for estimation of the DNA content for each chromosome. One was based on the relative length of each bivalent, but the DNA content would be underestimated for relatively heterochromatic chromosomes and overestimated for relatively euchromatic chromosomes. The other was based on relative DAPI fluorescence measurements, which was considered to be more accurate because it 
took not only the relative length of each bivalent but also the density into account.

Integration of cytogenetic and genetic maps in potato

While linkage maps accurately describe gene order and the frequency of crossovers between genes or markers, linkage map distances are not simply related to physical distances (Harper and Cande 2000; Anderson et al. 2004). This is because crossovers are not equally distributed over chromosome arms, and as a result loci that are physically far apart on chromosomes can be tightly linked on linkage maps and vice versa (Islam-Faridi et al. 2002; Budiman et al. 2004). Such discrepancies are impediments to applying linkage maps to guide genome sequence assembly or for gene discovery by chromosome walking.

To display the relationship between the genetic and chromosome map in potato, we calculated the ratio of genetic distance (bin) to cytological distance (FL) between pairs of BAC markers. The ratio varies from $3.90 \mathrm{bin} / \mathrm{FL}(22 \mathrm{bins} / 5.65 \mathrm{FL})$ in the distal region of 7L (long arm of chromosome 7) to $0.18 \mathrm{bin} / \mathrm{FL}$ (six bins/33.19 FL) in the pericentromere of chromosome 9, which corresponds to $6.27 \mathrm{cM} / \mu \mathrm{m}$ for $7 \mathrm{~L}$ and $0.22 \mathrm{cM} / \mu \mathrm{m}$ for the pericentromere region of chromosome 9, respectively. These values demonstrate that the recombination frequency in the distal region of the chromosome is much higher than that in the pericentromere region (Table 1). To better estimate the suppression of recombination in the pericentromere region, we performed FISH hybridizations on chromosome 5. In an interval of approximately 21.78 $\mu \mathrm{m}$ between clones RH094I11 and RH071D16, the genetic map shows no recombination since all markers in this region belong to a single bin (bin046). This means that the recombination frequency in this region must be less than one bin $(0.77 \mathrm{cM})$ per $21.78 \mu \mathrm{m}$, which is less than $0.04 \mathrm{cM} / \mu \mathrm{m}$.

Our range of recombination densities observed in potato, including the level of suppression in the pericentromere region, is comparable to the chromosomes in crops with a similar or larger genome size like maize, wheat, barley, and sorghum (Kim et al. 2005; Wang et al. 2006a). For instance, in maize chromosome 9, the recombination rate around the centromere region was highly decreased by 145 -fold from $7.29 \mathrm{cM} / \mu \mathrm{m}$ near the tip of $9 \mathrm{~S}$ to $0.05 \mathrm{cM} / \mu \mathrm{m}$ in the pericentromere region (Wang et al. 2006a).
Future applications of the potato cytogenetic map

Our current cytogenetic map forms a solid foundation for future FISH-based chromosome research in potato. The pachytene stage of meiosis offers the best cell material to perform such experiments, since it allows cytogenetic mapping at high resolution and gives a clear distinction of euchromatin and heterochromatin features (de Jong et al. 1999). A range of applications can be envisioned, such as development of a high-density cytogenetic map by the inclusion of additional probes, mapping of unanchored BAC clones or gene sequences, ordering of physical map contigs in low recombination regions, or examining the distribution pattern of repetitive sequences.

The reference set of 60 BACs can serve as a universal set of cytogenetic markers for the Solanaceae, since most of them come from euchromatin where most genes are located. It was demonstrated in rice and maize that gene sequences and order are generally well conserved in cultivars and related species and that gene-rich probes give strong FISH signals across genomes (Tang et al. 2007; Lamb et al. 2007). Therefore, this set of cytogenetic markers in potato is useful as a starting point to study synteny and chromosomal rearrangements between Solanaceae taxa and cultivars (Tanksley et al. 1992; Wu et al. 2009). Actually, our probes were demonstrated to produce signals in several clones of potato and two tomatoes, and BAC-FISH has already fulfilled to reveal paracentric inversions between potato and tomato for both chromosomes 5 and 6 (Iovene et al. 2008; Tang et al. 2008; Achenbach et al. 2009).

With the advance of genome research, cytogenetic maps will not only be valuable for integrating and organizing genetic, molecular, and cytological information but they will also provide a unique insight into genome and chromosome organization, meiotic disturbances in homologous pairing, and chromosome transmission. A complete sequence of the potato genome will be publicly available in the near future. Identification of euchromatin and heterochromatin at both the cytological and DNA sequence level will be a critical component for characterization of the potato genome. The further FISH-based localization of BACs and other sequences on the potato genome will be a significant advancement of Solanaceae genomics in general and will provide a very valuable support to the International Potato Genome-Sequencing Project. 
Open Access This article is distributed under the terms of the Creative Commons Attribution Noncommercial License which permits any noncommercial use, distribution, and reproduction in any medium, provided the original author(s) and source are credited.

\section{References}

Achenbach UC, Tang X, de Jong H, Gebhardt C (2009) Comparison of the chromosome maps around a resistance hot spot on chromosome $\mathrm{V}$ of potato and tomato using BAC-FISH painting. Genome (in press)

Anderson LK, Salameh N, Bass HW et al (2004) Integrating genetic linkage maps with pachytene chromosome structure in maize. Genetics 166:1923-1933

Barton DW (1950) Pachytene chromosome morphology of tomato chromosome complement. Am J Bot 37:639-643

Bennett MD, Cox AV, Leitch IJ (1997) Angiosperm DNA C-values database. http://www.rbgkew.org.uk/cval/databasel.html.

Bonierbale MW, Plaisted RL, Tanksley SD (1988) RFLP maps based on a common set of clones reveal modes of chromosomal evolution in potato and tomato. Genetics 120:1095-1103

Borm TJA (2008) Construction and use of a physical map of potato. Ph.D. Thesis, Wageningen UR, The Netherlands

Bradshaw JE, Pande B, Bryan GJ et al (2004) Interval mapping of quantitative trait loci for resistance to late blight [Phytophthora infestans (Mont.) de Bary], height and maturity in a tetraploid population of potato (Solanum tuberosum subsp. tuberosum). Genetics 168:983-995

Budiman MA, Chang SB, Lee S et al (2004) Localization of jointless-2 gene in the centromeric region of tomato chromosome 12 based on high resolution genetic and physical mapping. Theor Appl Genet 108:90-196

Chang SB, Yang TJ, Datema E et al (2008) FISH mapping and molecular organization of the major repetitive sequences of tomato. Chromosome Res 16(7):919-933

Cheng Z, Presting GG, Buell CR, Wing RA, Jiang J (2001a) High resolution pachytene chromosome mapping of bacterial artificial chromosomes anchored by genetic markers reveals the centromere location and the distribution of genetic recombination along chromosome 10 of rice. Genetics 157:1749-1757

Cheng Z, Buell CR, Wing RA, Gu M, Jiang J (2001b) Toward a cytological characterization of the rice genome. Genome Res 11:2133-2141

de Jong JH, Fransz P, Zabel P (1999) High resolution FISH in plants - techniques and applications. Trends Plant Sci 4:258-263

Dong F, Song J, Naess SK, Helgeson JP, Gebhardt C, Jiang J (2000) Development and applications of a set of chromosome-specific cytogenetic DNA markers in potato. Theor Appl Genet 101:1001-1007

Dong F, McGrath JM, Helgeson JP, Jiang J (2001) The genetic identity of alien chromosomes in potato breeding lines revealed by sequential GISH and FISH analyses using chromosome-specific cytogenetic DNA markers. Genome 44:729-734
Dong F, Tek AL, Frasca ABL et al (2005) Development and characterization of potato-Solanum brevidens chromosomal addition/substitution lines. Cytogenet Genome Res 109:368-372

Fransz P, Armstrong S, Alonso-Blanco C, Fischer TC, TorresRuiz RA, Jones G (1998) Cytogenetics for the model system Arabidopsis thaliana. Plant J 13:867-876

Fransz PF, Armstrong S, de Jong JH et al (2000) Integrated cytogenetic map of chromosome arm $4 \mathrm{~S}$ of $A$. thaliana: structural organization of heterochromatic knob and centromere region. Cell 100:367-376

Fuchs J, Kloos DU, Ganal MW, Schubert I (1996) In situ localization of yeast artificial chromosome sequences on tomato and potato metaphase chromosomes. Chromosome Res 4:277-281

Gebhardt C, Ritter E, Barone A et al (1991) RFLP maps of potato and their alignment with the homeologous tomato genome. Theor Appl Genet 83:49-57

Harper LC, Cande WZ (2000) Mapping a new frontier; development of integrated cytogenetic maps in plants. Funct Integr Genomics 1:89-98

Hermsen JGT, Taylor LM, van Breukelen EWM, Lipski A (1978) Inheritance of genetic markers from two potato dihaploids and their respective parent cultivars. Euphytica 27:681-688

Iovene M, Wielgus SM, Simon PW, Buell CR, Jiang J (2008) High resolution fluorescence in situ hybridization mapping of chromosome 6 of potato and comparative analyses with tomato. Genetics 180:1307-1317

Islam-Faridi MN, Childs KL, Klein PE et al (2002) A molecular cytogenetic map of sorghum chromosome 1: fluorescence in situ hybridization analysis with mapped bacterial artificial chromosomes. Genetics 161:345-353

Kim JS, Childs KL, Islam-Faridi MN et al (2002) Integrated karyotyping of sorghum by in situ hybridization of landed BACs. Genome 45:402-412

Kim JS, Islam-Faridi MN, Klein PE et al (2005) Comprehensive molecular cytogenetic analysis of sorghum genome architecture: distribution of euchromatin, heterochromatin, genes and recombination in comparison to rice. Genetics 171:1963-1976

Kloosterman B, de Koeyer D, Griffiths R et al (2008) Genes driving potato tuber initiation and growth: identification based on transcriptional changes using the POCI array. Funct Integr Genomics 8:329-340

Kocsis E, Trus BL, Steer CJ, Bisher ME, Steven AC (1991) Image averaging of flexible fibrous macromolecules: the clathrin triskelion has an elastic proximal segment. J Struct Biol 107:6-14

Koo DH, Jo SH, Bang JW, Park HM, Lee S, Choi D (2008) Integration of cytogenetic and genetic linkage maps unveils the physical architecture of tomato chromosome 2. Genetics 179:1211-1220

Kulikova O, Gualtieri G, Kim DJ, Cook D, Huguet T, Bisseling T (2001) Integration of the FISH pachytene and genetic maps of Medicago truncatula. Plant J 27:49-58

Lamb JC, Danilova T, Bauer MJ et al (2007) Single-gene detection and karyotyping using small-target fluorescence in situ hybridization on maize somatic chromosomes. Genetics 175:1047-1058

Lapitan NLV, Brown SE, Kennard W, Stephens JL, Knudson DL (1997) FISH physical mapping with barley BAC clones. Plant J 11:149-156 
Levan A, Fredga K, Sandberg A (1964) Nomenclature for centromeric position on chromosomes. Hereditas 52:201-220

Nagaki K, Cheng Z, Ouyang S et al (2004) Sequencing of a rice centromere uncovers active genes. Nature genetics 36 (2):138-145

Nielsen KL, Gronkjaer K, Welinder KG, Emmersen J (2005) Global transcript profiling of potato tuber using LongSAGE. Plant Biotechnol J 3:175-185

Park T-H, Kim J-B, Hutten RCB, van Eck HJ, Jacobsen E, Visser RGF (2007) Genetic positioning of centromeres using Half-Tetrad analysis in a $4 x-2 x$ cross population of potato. Genetics 176:85-94

Peterson DG, Price HJ, Johnston JS, Stack SM (1996) DNA content of heterochromatin and euchromatin in tomato (Lycopersicon esculentum) pachytene chromosomes. Genome 39:77-82

Pijnacker LP, Ferwerda MA (1984) Giemsa C-banding of potato chromosomes. Can J Genet Cytol 26:415-419

Ramanna MS, Wagenvoort M (1976) Identification of the trisomic series in diploid Solanum tuberosum L., group tuberosum. I. Chromosome identification. Euphytica 25:233-240

Rensink WA, Lee Y, Liu J, Iobst S, Ouyang S, Buell CR (2005) Comparative analyses of six solanaceous transcriptomes reveal a high degree of sequence conservation and speciesspecific transcripts. BMC Genomics 6:124

Richards EJ, Ausubel FM (1988) Isolation of a higher eukaryotic telomere from Arabidopsis thaliana. Cell 53:127-136

Rouppe van der Voort JN, van Zandvoort P, van Eck HJ et al (1997) Use of allele specificity of comigrating AFLP markers to align genetic maps from different potato genotypes. Mol Gen Genet 255:438-447

Sherman JD, Stack SM (1995) Two-dimensional spreads of synaptonemal complexes from solanaceous plants. VI. High-resolution recombination nodule map for tomato (Lycopersicon esculentum). Genetics 141:683-708

Szinay D, Chang SB, Khrustaleva L et al (2008) Highresolution chromosome mapping of BACs using multicolour FISH and pooled-BAC FISH as a backbone for sequencing tomato chromosome 6. Plant J 56(4):627-637

Tang X, Bao W, Zhang W, Cheng Z (2007) Identification of chromosomes from multiple rice genomes using a universal molecular cytogenetic marker system. J Integr Plant Biol 49(6):953-960

Tang X, Szinay D, Lang C et al (2008) Cross-species BACFISH painting of the tomato and potato chromosome 6 reveals undescribed chromosomal rearrangements. Genetics 180:1319-1328

Tanksley SD, Ganal MW, Prince JP et al (1992) High density molecular linkage maps of the tomato and potato genomes. Genetics 132:1141-1160

Tek AL, Stevenson WR, Helgeson JP, Jiang J (2004) Transfer of tuber soft rot and early blight resistances from Solanum brevidens into cultivated potato. Theor Appl Genet 109:249-254

van Eck HJ, van der Voort JR, Draaistra J et al (1995) The inheritance and chromosomal localization of AFLP markers in a non-inbred potato offspring. Mol Breeding 1:397-410 van Os H, Andrzejewski S, Bakker E et al (2006) Construction of a 10, 000-marker ultra-dense genetic recombination map of potato: providing a framework for accelerated gene isolation and a genome wide physical map. Genetics 173:1075-1087

Visser RGF, Hoekstra R, Vander L, Pijnacker LP, Witholt B, Feenstra WJ (1988) In situ hybridization to somatic metaphase chromosomes of potato. Theor Appl Genet 76:420-424

Visser RGF, Bachem CWB, de Boer JM et al (2009) Sequencing the potato genome: outline and first results to come from the elucidation of the sequence of the world's third most important food crop. Am J Potato Res (in press). doi:10.1007/s12230-009-9097-8

Wagenvoort M (1988) Spontaneous structural rearrangements in Solanum tuberosum L group Tuberosum II Identification at pachytene stage. Euphytica 39:159-167

Wagenvoort M, Rouwendal GJA, Kuiper-Groenwold G, de Vries-van Hulten HPJ (1994) Chromosome identification in potato trisomics $(2 n=2 x+1=25)$ by conventional staining, Giemsa C-banding and non-radioactive in situ hybridization. Cytologia 59:405-417

Wang C-JR, Harper L, Cande WZ (2006a) High-resolution single-copy gene fluorescence in situ hybridization and its use in the construction of a cytogenetic map of maize chromosome 9. The Plant Cell 18:529-544

Wang Y, Tang X, Cheng Z, Mueller L, Giovannoni J, Tanksley SD (2006b) Euchromatin and pericentromeric heterochromatin: comparative composition in the tomato genome. Genetics 172:2529-2540

Wu J, Yamagata H, Hayashi-Tsugane M et al (2004) Composition and structure of the centromeric region of rice chromosome 8. The Plant Cell 16:967-976

Wu F, Eannetta NT, Xu Y, Tanksley SD (2009) A detailed synteny map of the eggplant genome based on conserved ortholog set II (COSII) markers. Theor Appl Genet 118:927-935

Yan H, Jin W, Nagaki K et al (2005) Transcription and histone modifications in the recombination-free region spanning a rice centromere. The Plant Cell 17:3227-3238

Yeh BP, Peloquin SJ (1965) Pachytene chromosomes of the potato (Solanum tuberosum, Group Andigena). Am J Bot 52:1014-1020

Zhao Q, Zhang Y, Cheng Z et al (2002) A fine physical map of the rice chromosome 4. Genome Res 12:817-823

Zhong XB, de Jong JH, Zabel P (1996a) Preparation of tomato meiotic pachytene and mitotic metaphase chromosomes suitable for fluorescence in situ hybridisation (FISH). Chromosome Res 4:24-28

Zhong XB, Fransz PF, van Eden JW, Zabel P, van Kammen A, de Jong JH (1996b) High resolution mapping by fluorescence in situ hybridisation to pachytene chromosomes and extended DNA fibres. Plant Mol Biol Rep $14: 232-242$

Zhong XB, Bodeau J, Fransz PF et al (1999) FISH to meiotic pachytene chromosomes of tomato locates the root knot nematode resistance gene $M i-1$ and the acid phosphatase gene Aps-1 near the junction of euchromatin and pericentromeric heterochromatin of chromosome arms $6 \mathrm{~S}$ and $6 \mathrm{~L}$, respectively. Theor Appl Genet 98: $365-370$ 